\title{
Effect of the prognostic nutritional index on prognosis in patients undergoing gastric cancer surgery
}

\author{
Aziz Serkan Senger ${ }^{1}$, Selcuk Gulmez ${ }^{1}$,Orhan Uzun ${ }^{1}$, Deniz Avan ${ }^{2}$, Ayhan Oz ${ }^{1}$, Cem B. Ofluoglu ${ }^{1}$, Omer Ozduman ${ }^{1}$, \\ Erdal Polat ${ }^{1}$, Mustafa Duman ${ }^{1}$ \\ ${ }^{1}$ Department of Gastrointestinal Surgery, Kartal Kosuyolu Higher Specialty Training and Research Hospital, University of Health Sciences, Istanbul, Turkey \\ ${ }^{2}$ Department of Anesthesia and Reanimation Clinic, Kartal Kosuyolu Higher Specialty Training and Research Hospital, University of Health Sciences, Istanbul, \\ Turkey
}

Received: 2020-11-14.

Accepted: 2021-01-12

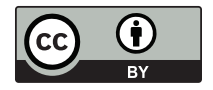

This work is licensed under a Creative Commons Attribution 4.0 International License

J Clin Med Kaz 2021; 18(1):69-73

Corresponding author:

Aziz Serkan Senger.

E-mail: serkansenger@yahoo.com;

\section{Abstract}

Objective: The leading concern among cancer patients is posttreatment complications and survival, which are associated with patient-related, tumor-related and treatment-related factors. We present here an analysis of the Prognostic Nutritional Index as a preoperatively improvable prognostic factor.

Material and methods: A retrospective review was made of 228 patients operated on for gastric cancer between January 2011 and October 2019. The patients were divided into two groups based on Prognostic Nutritional Index (PNI <45 and PNI $\geq 45$ ). The clinicopathological characteristics and prognosis of the patients were recorded and analyzed.

Results: Prognostic Nutritional Index was $\geq 45$ in 162 patients and $<45$ in 66 patients. An examination of the patients' clinicopathological and demographic characteristics revealed a statistically significant difference in postoperative complications $(p=0.014)$, age $(p=0.054)$, tumor size ( $p=0.027)$, and Body Mass Index (BMI) $(p=0.041)$ between the two groups. Using a Kaplan-Meier test, overall survival analysis revealed a difference between the two groups $(p=0.001)$. A multivariate Cox regression analysis of the factors impacting overall survival revealed the T stage $(p=0.031)$, $N$ stage $(p<0.001)$, and PNI $(p=0.041)$ to be significant.

Conclusion: The prognostic nutritional index is easy to calculate, a simple and practical approach to assessing nutritional status. Based on the present study's findings, the preoperative calculation of the prognostic nutritional index can serve as an indicator of postoperative complications and prognosis. The most important benefit of this marker is that it can be improved preoperatively.

Key words: Prognostic Nutritional Index, gastric cancer, prognosis, complications

\begin{abstract}
Introduction
More than 1 million gastric cancer cases are diagnosed globally every year. Gastric cancer is the third leading cause of cancer-related death [1]. The optimum treatment approach to locally advanced gastric cancer is still surgery [2], while chemotherapy and radiotherapy are other treatment options, being used both preoperatively and postoperatively. Five-year survival is 20-30\% with surgical resection alone, while survival outcomes have been observed to improve with the addition of chemotherapy-radiotherapy to the treatment [3]. Despite advances in surgical techniques and perioperative care, the rate of complications after gastrectomy is still in the $10.5-40.1 \%$ range [4].
\end{abstract}

Malnutrition is one of the leading factors facilitating complications after surgical intervention. According to the definition by the World Health Organization announced in 2020, malnutrition refers to deficiencies, excesses, or imbalances in an individual's intake of energy and nutrients. The incidence of malnutrition among cancer patients is $40-80 \%$ and varies in accordance with the type, localization, and stage of the tumor [5]. Malnourished patients are reported to be at increased risk of infection, reduced adherence to anti-cancer treatment and quality of life, and increased morbidity and mortality rates. That said, weight loss, low Body Mass Index (BMI), and low albumin levels may not always be associated with morbidity and mortality $[6,7]$. 
The Prognostic Nutritional Index (PNI) is a tool for measuring the nutritional and immunological status and is calculated based on serum albumin concentration and total lymphocyte count [8]. PNI is associated with mortality and prognosis in cancer patients and has been shown to predict postoperative complications following gastrointestinal system surgery $[9,10]$.

The present study assesses the relationship between PNI, complications, and overall survival among patients operated on for gastric cancer.

\section{Material and methods Patients}

The study involved a retrospective examination of 228 patients operated on for gastric cancer in the XXX Hospital between January 2011 and October 2019. Patients whose gastric biopsy is adenocarcinoma, who underwent a curative gastrectomy and D2 lymph node dissection were included in the study, while those who underwent emergency surgery or palliative resection, with no accessible records and with distant metastasis were excluded. Ethical approval (Ethical Committee No. 2019.7/43-259) was provided by the Institutional Research and Ethics Committee of our hospital.

\section{Data}

Demographic and clinicopathological characteristics were collected retrospectively from medical records and analyzed, including the patients' age, gender, BMI, tumor localization, tumor size and histology, $\mathrm{T}$ stage, $\mathrm{N}$ stage, the total number of lymph nodes, TNM stage, vascular invasion, lymphatic invasion, neoadjuvant therapy, type of operation and postoperative complications [11].

The gastric cancer classification was made in accordance with the UICC/AJCC 8th Edition [12]. Serum albumin (g/ dl) and total lymphocyte counts (per mm3) measure from preoperativelycollected serum samples were recorded. PNI was calculated using the following formula: [serum albumin in $\mathrm{g} / \mathrm{dL} \times 10]+[0.005 \times$ total lymphocyte count in cells $/ \mu \mathrm{L}]$. Postoperative complications were examined retrospectively and recorded. Those occurring within the postoperative first 30 days or before discharge in the event of a longer hospital stay were considered postoperative complications. Based on previous studies, the cutoff value of PNI was set at 45 , and the patients were duly divided into groups: PNI $<45$ and PNI $\geq 45$ [13]. The date for the last follow-up visit of patients was taken as December 31, 2019.

\section{Statistical analysis}

The IBM SPSS Statistics for Windows (IBM Corp. Released 2013. IBM SPSS Statistics for Windows, Version 22.0. Armonk, NY: IBM Corp.) was used for the study's statistical analyses. The normality of the data was analyzed with a Kolmogorov-Smirnov test. Qualitative data were presented as frequency and percentage, and quantitative data were expressed as mean $\pm \mathrm{SD}$ if the data were normally distributed and as median (Interquartile range - IQR) if not normally distributed. The differences between the prognostic nutritional index and the categorical variables was analyzed using Chi-square and Fisher's exact tests. A Mann-Whitney U-test was used to identify any relationship between tumor size and BMI and PNI. The age and PNI relationship, in turn, was analyzed using a Student's t-test. A multivariate analysis was carried out using a Cox regression analysis to assess the prognostic factors for survival,includingage, gender, tumor localization, signet-ring cell histology, tumor depth of wall invasion, tumor size, lymph node status, presence of complications, PNI status, vascular invasion, and perineural invasion. A p-value lower than 0.05 was accepted as statistically significant.

\section{Results}

PNI was $\geq 45$ in $162(71.05 \%)$ of the 228 patients. An examination of the two groups revealed no statistically significant difference in age, neoadjuvant therapy, signet-ring cell histology, tumor localization, type of operation, the total number of lymph nodes removed, tumor depth of wall invasion, stage, vascular invasion, and perineural invasion $(\mathrm{p}>0.05)$. There was a statistically significant difference in tumor size between the two groups $(\mathrm{p}=0.027)$, with the low PNI group reporting greater tumor sizes. There was also a statistically significant difference in BMI between the two groups $(\mathrm{p}=0.041)$, and the group with a higher BMI also had a higher PNI value. A comparison of complications between the two groups revealed a PNI of $<45$ in $43.9 \%$ and $\geq 45$ in $27.2 \%$ of the patients who developed complications $(\mathrm{p}=0.014)$ (Table 1$)$.

There was a statistically significant difference in overall survival between the two groups $(\mathrm{p}=0.001)$ (Figure 1). The mean survival of patients with PNI $<45$ was 39.0 months, compared to 87.1 months in those with PNI $\geq 45$ (Table 2).

A multivariate Cox regression analysis was made to assess the effect of prognostic factors on overall survival. Tumor depth of wall invasion $(p=0.031)$, lymph node involvement $(p<0.001)$ and PNI $(p=0.041)$ were identified as prognostic factors. The mortality rate was 1.5 times higher in patients with $\mathrm{PNI}<45$ than in the other group. Likewise, the mortality rate was 4.2 times higher among $\mathrm{pN} 3$-stage patients than $\mathrm{pN} 0$ patients $(\mathrm{p}<0.001)$, and similarly a 3.5 times higher rate among $\mathrm{pN} 1$ patients than pN0 patients $(\mathrm{p}=0.002)$ (Table 3$)$.

\section{Discussion}

The aim of this study was to investigate the relationship between the preoperative prognostic nutritional index value and postoperative outcomes.

Gastric cancer is a common disease associated with a poor prognosis and is among the leading causes of cancer-related death. There have been papers reporting postoperative complications to significantly affect overall survival, and others reported no effect of complications and sepsis on oncological outcomes [1315]. It is critically important to identify preoperative risk factors for improved outcomes [16]. Certain risk factors related to the surgical team, patient, and disease can be identified and improved before surgery. Nutritional status is a patient-related factor that can be determined preoperatively, and malnutrition may ocur due to insufficient food intake, imbalanced nutrition,or an excessively increased catabolism, despite adequate food intake [17]. Several methods have been developed for the assessment of malnutrition, including anthropometric measurements, the Nutritional Risk Index (NRI), Nutritional Risk Screening (NRS2002), Subjective Global Assessment (SGA), Mini Nutritional Assessment (MNA), the Charlson comorbidity index (CCI), NUTRIC score and specific biochemical parameters [18].

PNI, which is calculated based on two routine laboratory parameters, is both objective and easily calculable, in which albumin level reflects the patient's nutritional status, while lymphocytes provide information on their immunological status [9].

Journal of Clinical Medicine of Kazakhstan: 2021 Volume 18, Issue 1 


\begin{tabular}{|c|c|c|c|c|}
\hline \multicolumn{2}{|l|}{ Variables } & \multirow{2}{*}{$\begin{array}{l}\text { PNI } \geq 45 \\
n=66(28.9 \%) \\
42(63.6 \%) \\
\end{array}$} & \multirow{2}{*}{$\begin{array}{l}\text { PNI <45 } \\
n=162(71.1 \%) \\
114(70.4 \%)\end{array}$} & \multirow{2}{*}{$\begin{array}{l}\text {-value } \\
0.321 \\
\end{array}$} \\
\hline Gender & Male & & & \\
\hline & Female & $24(36.4 \%)$ & $48(29.6 \%)$ & \\
\hline \multirow[t]{3}{*}{ Tumor location } & Upper $1 / 3$ & $15(22.7 \%)$ & $38(23.5 \%)$ & 0.569 \\
\hline & Middle 1/3 & $22(33.3 \%)$ & $43(26.5 \%)$ & \\
\hline & Lower $1 / 3$ & $29(43.9 \%)$ & $81(50.0 \%)$ & \\
\hline \multirow[t]{2}{*}{ Tumor histology } & Other & $47(71.2 \%)$ & $113(69.8 \%)$ & 0.827 \\
\hline & Signet-cell & $19(28.8 \%)$ & $49(30.2 \%)$ & \\
\hline \multirow[t]{2}{*}{ Neoadjuvant therapy } & No & $54(81.8 \%)$ & $128(79.0 \%)$ & 0.632 \\
\hline & Yes & $12(18.2 \%)$ & $34(21.0 \%)$ & \\
\hline \multirow[t]{2}{*}{ Type of operation } & Subtotal & $32(48.5 \%)$ & $77(47.5 \%)$ & 0.896 \\
\hline & Total & $34(51.5 \%)$ & $85(52.5 \%)$ & \\
\hline \multirow[t]{3}{*}{ pT stage } & pT1 & $8(12.1 \%)$ & $20(12.3 \%)$ & 0.302 \\
\hline & pT2 & $7(10.6 \%)$ & $20(12.3 \%)$ & \\
\hline & pT4 & $23(34.8 \%)$ & $37(22.8 \%)$ & \\
\hline \multirow[t]{4}{*}{ pN stage } & $\mathrm{pN} 0$ & $20(30.3 \%)$ & $59(36.4 \%)$ & 0.769 \\
\hline & $\mathrm{pN} 1$ & $13(19.7 \%)$ & $27(16.7 \%)$ & \\
\hline & $\mathrm{pN} 2$ & $10(15.2 \%)$ & $27(16.7 \%)$ & \\
\hline & $\mathrm{pN} 3$ & $23(34.8 \%)$ & $49(30.2 \%)$ & \\
\hline \multirow[t]{3}{*}{ TNM Stage } & I & $14(21.2 \%)$ & $25(15.4 \%)$ & 0.191 \\
\hline & II & $14(21.2 \%)$ & $53(32.7 \%)$ & \\
\hline & III & $38(57.6 \%)$ & $84(51.9 \%)$ & \\
\hline \multirow[t]{2}{*}{ Vascular invasion } & No & $26(39.4 \%)$ & $68(42.0 \%)$ & 0.720 \\
\hline & Yes & $40(60.6 \%)$ & $94(58.0 \%)$ & \\
\hline \multirow[t]{2}{*}{ Perineural invasion } & No & $25(37.9 \%)$ & $58(35.8 \%)$ & 0.768 \\
\hline & Yes & $41(62.1 \%)$ & $104(64.2 \%)$ & \\
\hline Postoperative complications & No & $37(56.1 \%)$ & $118(72.8 \%)$ & $0.014 \mathrm{a}$ \\
\hline Age, years & & $62 \pm 11$ & $59 \pm 11$ & $0.054 \mathrm{~b}$ \\
\hline Total number of lymph nodes & & $24(9-57)$ & $26(8-74)$ & 0.531 \\
\hline Tumor size & & $5.5(0.7-13)$ & $4.5(0.5-18)$ & $0.027 \mathrm{c}$ \\
\hline Body Mass Index & & $24.90(16.26-37.89)$ & 26.75 (15.4-44.12) & $0.041 \mathrm{c}$ \\
\hline
\end{tabular}

aChi-square test; bStudents' t test; cMann-Whitney U-test; Standard Deviation

Figure 1 - Overall survival analysis by Kaplan-Meier test according to PNI

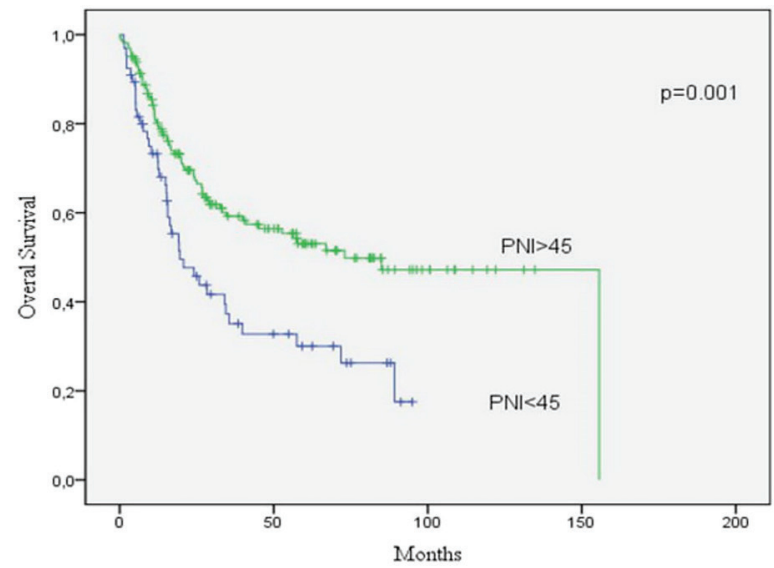

Table 2

Comparison of overall survival for PNI based on a Kaplan-Meier test

\begin{tabular}{|l|l|l|l|l|}
\hline PNI & Estimate & Std. Error & $95 \%$ CI & p-value \\
\hline$<45$ & 39,015 & 4,889 & $29,433-$ & 0.001 \\
& & & 48,598 & \\
\hline$\geq 45$ & 87,133 & 6,167 & $75,046-$ & \\
& & & 99,220 & \\
\hline
\end{tabular}

PNI: Prognostic Nutritional Index; CI: Confidence Interval
Table 3

Evaluation of the effect of prognostic factors on overall survival based on a multivariate Cox regression analysis

\begin{tabular}{llll}
\hline Variables & OR & $95.0 \%$ CI & p-value \\
\hline Age, years & 1.013 & $0.994-1.033$ & 0.173 \\
Gender, female & 0.788 & $0.494-1.256$ & 0.316 \\
Tumor location (upper third) & 1 & & 0.248 \\
Tumor location (middle third) & 1.553 & $0.810-0.602$ & 0.185 \\
Tumor location (lower third) & 1.090 & $2.979-1.973$ & 0.775 \\
Signet-ring cell & 1.252 & $0.807-1.941$ & 0.315 \\
pT1 & 1 & & 0.031 \\
pT2 & 0.750 & $0.169-0.466$ & 0.705 \\
pT3 & 1.791 & $0.723-3.326$ & 0.396 \\
pT4 & 2.956 & $6.878-12.081$ & 0.131 \\
Tumor size & 1.028 & $0.948-1.115$ & 0.506 \\
pN0 & 1 & & $<0.001$ \\
pN1 & 3.523 & $1.571-7.901$ & 0.002 \\
pN2 & 3.942 & $1.676-8.655$ & 0.001 \\
pN3 & 4.217 & $1.896-9.378$ & $<0.001$ \\
Postoperative complications & 1.541 & $0.993-2.393$ & 0.054 \\
Prognostic nutritional index, $\geq 45$ & 0.642 & $0.420-0.982$ & 0.041 \\
Vascular invasion & 0.686 & $0.399-1.179$ & 0.173 \\
Perineural invasion & 1.389 & $0.821-2.352$ & 0.221
\end{tabular}

OR: Odds Ratio; CI: Confidence Interval

The primary treatment modality for gastric cancer is surgery, and depending on the stage and localization, perioperative or postoperative chemotherapy will also be required. Postoperative complications can lead to a delay in oncological treatment. It can 
be attributed to several factors, including duration of operation, type of operation (emergency or elective), age, comorbidities, and nutritional status. Aside from these, BMI has also been reported as a potential risk factor for complication development. Toshimasa T. et al. reported more frequent complications among postoperative patients with a BMI $\geq 25$ [19], while in the study by Fan Feng et al., a low BMI was reported to havenoimpact on complications other than low postoperative fever, but negatively affected overall survival [20]. Similar to the study by Kamachi et al., the present study identified low BMI, like PNI, to be a predisposing factor for the development of complications.

The factors impacting overall survival in gastric cancer include tumor depth, lymph node involvement, and distant organ metastasis, all of which are used in staging [21]. Although tumor size is not included in staging, studies reported increased tumor size correlated with lymph node involvement and a significant prognostic factor [22]. When the risk factors for overall survival were examined in the present study, it was found that a low PNI had a negative effect on survival,along with an increased pathological $\mathrm{T}$ stage and pathological $\mathrm{N}$ stage.
The limitations of the present study include its retrospective design, its short follow-up period for some patients, and the absence of chemotherapy details of the patients.

In conclusion, it is important to establish the preoperative prognostic risk factors when determining the optimal treatment approach and improve postoperative short and long term outcomes. Accordingly, PNI should be calculated for patients scheduled for gastric cancer surgery, and those with a low PNI should be assessed for enteral or parenteral nutritional support. It is necessary to recommend further prospective studies to prove the effectiveness of PNI correction before surgery for this category of patients.

Disclosures: There is no conflict of interest for all authors.

Funding: None.

\section{References}

1. Kopp I, Bauhofer A, Koller M. Understanding quality of life in patients with colorectal cancer: comparison of data from a randomised

2. Santos, M.O., \& Silva, J.G. (2018). Estimate 2018: Cancer Incidence in Brazil Estimativa 2018: Incidência de Câncer no Brasil Estimación 2018 : incidencia de Cáncer en brasil.

3. Kamiya S, Rouvelas I, Lindblad M, Nilsson M. Current trends in gastric cancer treatment in Europe. J Cancer Metastasis Treat. 2018; 4(7):35. http://dx.doi.org/10.20517/2394-4722.2017.76.

4. Yaprak G, Tataroglu D, Dogan B, Pekyurek M. Prognostic factors for survival in patients with gastric cancer: single instution experience. North Clin Istanbul. 2019; 7(2):146-52. doi: 10.14744/nci.2019.73549

5. Zhou J, Hiki N, Mine S, Kumagai K, Ida S, Jiang X, et al. Role of Prealbumin as a Powerful and Simple Index for Predicting Postoperative Complications After Gastric Cancer Surgery. Ann Surg Oncol. 2017; 24(2):510-7. doi:10.1245/s10434-016-5548-x

6. Lis CG, Gupta D, Lammersfeld CA, Markman M, Vashi PG. Role of nutritional status in predicting quality of life outcomes in cancer - A systematic review of the epidemiological literature. Nutr J. 2012; 11(1). doi:10.1186/1475-2891-11-27

7. Leandro-Merhi VA, de Aquino JLB. Determinants of malnutrition and post-operative complications in hospitalized surgical patients. J Heal Popul Nutr. 2014; 32(3):400-10. https://doi.org/10.1016/S0261-5614(13)60457-4

8. Claudino MM, Lopes JR, Rodrigues VD, de Pinho NB, Martucci RB. Postoperative complication rate and survival of patients with gastric cancer undergoing immunonutrition: A retrospective study. Nutrition [Internet]. 2020; 70:110590. https://doi.org/10.1016/j. nut.2019.110590.

9. Okadome K, Baba Y, Yagi T, Kiyozumi Y, Ishimoto T, Iwatsuki M, et al. Prognostic Nutritional Index, Tumor-infiltrating Lymphocytes, and Prognosis in Patients with Esophageal Cancer. Ann Surg. 2020; 271(4):693-700. doi: 10.1097/SLA.0000000000002985.

10. Miao Y, Li S, Yan Q, Li B, Feng Y. Prognostic significance of preoperative prognostic nutritional index in epithelial ovarian cancer patients treated with platinum-based chemotherapy. Oncol Res Treat. 2016; 39(11):712-9. https://doi.org/10.1159/000452263.

11. Luvián-Morales J, González-Trejo S, Carrillo JF, Herrera-Goepfert R, Aiello-Crocifoglio V, Gallardo-Rincón D, et al. Association of the prognostic nutritional index and overall survival in patients with colorectal cancer: A STROBE compliant retrospective cohort study. Cancer Med. 2019; 8(7):3379-88. https://doi.org/10.1002/cam4.2212.

12. Jiang N, Deng JY, Ding XW, Ke B, Liu N, Zhang RP, et al. Prognostic nutritional index predicts postoperative complications and longterm outcomes of gastric cancer. World J Gastroenterol. 2014; 20(30):10537-44. doi: 10.3748/wjg.v20.i30.10537.

13. Amin MB, Edge SB, Greene FL, Gershenwald JE, Brookland RK, Meyer L. et al. The AJCC Cancer Staging Manual, 8th ed.; Springer: New York, NY, USA, 2016.

14. Özbilgin Ş, Hanci V, Ömür D, Özbilgin M, Tosun M, Yurtlu S, et al. Morbidity and mortality predictivity of nutritional assessment tools in the postoperative care unit. Medicine. 2016; 95(40):1-7. http://dx.doi.org/10.1097/MD.0000000000005038.

15. Tsujinaka T, Sasako M, Yamamoto S, Sano T, Kurokawa Y, Nashimoto A, et al. Influence of overweight on surgical complications for gastric cancer: Results from a randomized control trial comparing D2 and extended para-aortic D3 lymphadenectomy (JCOG9501). Ann Surg Oncol. 2007; 14(2):355-61. doi: 10.1245/s10434-006-9209-3.

16. Feng F, Zheng G, Guo X, Liu Z, Xu G, Wang F, et al. Impact of body mass index on surgical outcomes of gastric cancer. BMC Cancer. 2018; 18(1):1-8. https://doi.org/10.1186/s12885-018-4063-9.

17. Kamachi K, Ozawa S, Hayashi T, Kazuno A, Ito E, Makuuchi H. Impact of body mass index on postoperative complications and longterm survival in patients with esophageal squamous cell cancer. Dis Esophagus. 2016; 29(3):229-35. doi: 10.1111/dote.12327.

18. Li C, Oh SJ, Kim S, Hyung WJ, Yan M, Zhu ZG, et al. Risk factors of survival and surgical treatment for advanced gastric cancer with large tumor size. J Gastrointest Surg. 2009; 13(5):881-5. doi: 10.1007/s11605-009-0800-3.

19. Zhao LY, Zhang WH, Chen XZ, Yang K, Chen XL, Liu K, et al. Prognostic significance of tumor size in 2405 patients with gastric cancer a retrospective cohort study. Medicine. 2015; 94(50):1-10. doi: 10.1097/MD.0000000000002288. 
20. Jiang N, Deng JY, Ding XW, Zhang L, Liu HG, Liang YX, et al. Effect of complication grade on survival following curative gastrectomy for carcinoma. World J Gastroenterol. 2014; 20(25):8244-52. DOI: 10.3748/wjg.v20.i25.8244.

21. Climent M, Hidalgo N, Vidal, Puig S, Iglesias M, Cuatrecasas M, et al. Postoperative complications do not impact on recurrence and survival after curative resection of gastric cancer. Eur J Surg Oncol. 2016; 42(1):132-9. http://dx.doi.org/10.1016/j.ejso.2015.08.163.

22. Wang S, Xu L, Wang Q, Li J, Bai B, Li Z, et al. Postoperative complications and prognosis after radical gastrectomy for gastric cancer: A systematic review and meta-analysis of observational studies. World J Surg Oncol. 2019; 17(1):18-20. https://doi.org/10.1186/s12957019-1593-9.

23. Argilés JM. Cancer-associated malnutrition. Eur J Oncol Nurs. 2005; 9(2). doi:10.1016/j.ejon.2005.09.006. 